\title{
C-reactive protein concentration as a risk predictor of mortality in intensive care unit: a multicenter, prospective, observational study
}

Rong $\mathrm{Qu}^{1,2+}$, Linhui Hu ${ }^{3,4+}$, Yun Ling ${ }^{2}$, Yating Hou ${ }^{4}$, Heng Fang ${ }^{5,6}$, Huidan Zhang ${ }^{5,6}$, Silin Liang ${ }^{5,6}$, Zhimei He ${ }^{6}$, Miaoxian Fang ${ }^{5}$, Jiaxin $\mathrm{Li}^{5}, \mathrm{Xu} \mathrm{Li}^{7^{*}}$ and Chunbo Chen ${ }^{1,8^{*}}$ (D)

\begin{abstract}
Background: It is not clear whether there are valuable inflammatory markers for prognosis judgment in the intensive care unit (ICU). We therefore conducted a multicenter, prospective, observational study to evaluate the prognostic role of inflammatory markers.

Methods: The clinical and laboratory data of patients at admission, including C-reactive protein (CRP), were collected in four general ICUs from September 1, 2018, to August 1, 2019. Multivariate logistic regression was used to identify factors independently associated with nonsurvival. The area under the receiver operating characteristic curve (AUC-ROC), net reclassification improvement (NRI), and integrated discrimination improvement (IDI) were used to evaluate the effect size of different factors in predicting mortality during ICU stay. 3 -knots were used to assess whether alternative cut points for these biomarkers were more appropriate.

Results: A total of 813 patients were recruited, among whom 121 patients (14.88\%) died during the ICU stay. The AUC-ROC values of PCT and CRP for discriminating ICU mortality were 0.696 (95\% confidence interval [CI], 0.650 $0.743)$ and $0.684(95 \% \mathrm{Cl}, 0.633-0.735)$, respectively. In the multivariable analysis, only APACHE II score (odds ratio, 1.166; 95\% Cl, 1.129-1.203; $P=0.000$ ) and CRP concentration $>62.8 \mathrm{mg} / \mathrm{L}$ (odds ratio, $2.145 ; 95 \% \mathrm{Cl}, 1.343-3.427 ; P=$ 0.001 ), were significantly associated with an increased risk of ICU mortality. Moreover, the combination of APACHE II score and CRP $>62.8 \mathrm{mg} / \mathrm{L}$ significantly improved risk reclassification over the APACHE II score alone, with NRI (0.556) and IDI (0.013). Restricted cubic spline analysis confirmed that CRP concentration $>62.8 \mathrm{mg} / \mathrm{L}$ was the optimal cut-off value for differentiating between surviving and nonsurviving patients.
\end{abstract}

Conclusion: CRP markedly improved risk reclassification for prognosis prediction.

Keywords: Procalcitonin, C-reactive protein, Intensive care unit, Biomarker, Mortality, Predictor

\footnotetext{
* Correspondence: mylx99@smu.edu.cn; gghccm@163.com

${ }^{\dagger}$ Rong Qu and Linhui Hu these authors contributed equally to this study, Cofirst author.

${ }^{7}$ State Key Laboratory of Organ Failure Research, Guangdong Provincial Key Laboratory of Viral Hepatitis Research, Department of Infectious Diseases, Nanfang Hospital, Southern Medical University, 1838 Guangzhou Avenue North, Guangzhou, Guangdong, China

'The Second School of Clinical Medicine, Southern Medical University, Guangzhou 510515, Guangdong, China

Full list of author information is available at the end of the article
}

(c) The Author(s). 2020 Open Access This article is licensed under a Creative Commons Attribution 4.0 International License, which permits use, sharing, adaptation, distribution and reproduction in any medium or format, as long as you give appropriate credit to the original author(s) and the source, provide a link to the Creative Commons licence, and indicate if changes were made. The images or other third party material in this article are included in the article's Creative Commons licence, unless indicated otherwise in a credit line to the material. If material is not included in the article's Creative Commons licence and your intended use is not permitted by statutory regulation or exceeds the permitted use, you will need to obtain permission directly from the copyright holder. To view a copy of this licence, visit http://creativecommons.org/licenses/by/4.0/ The Creative Commons Public Domain Dedication waiver (http://creativecommons.org/publicdomain/zero/1.0/) applies to the data made available in this article, unless otherwise stated in a credit line to the data. 


\section{Background}

Patients admitted to the ICU suffer from critical illness or injury and are at a high risk of dying. ICU mortality rates differ widely depending on the underlying disease process, with death rates as low as 1 in 20 for patients admitted following elective surgery and as high as 1 in 4 for patients with respiratory diseases [1]. The risk of death can be approximated by evaluating the severity of a patient's illness as determined by important pathophysiological, clinical, and demographic determinants. In clinical practice, estimates of mortality risk can be useful in resource allocation, in determining appropriate levels of care, and even in discussions with patients and their families about expected outcomes.

The use of clinical risk scores, such as the Acute Physiology and Chronic Health Evaluation II (APACHE II) score or the Sequential Organ Failure Assessment (SOFA) score $[2,3]$, despite their considerable prognostic accuracy for ICU mortality, is partly also limited by practicality issues. For instance, certain disease states or conditions may generate very high severity scores, even though they do not generally result in high mortality. These are usually conditions associated with a high degree of physiological derangement but which are either self-limiting or can be managed to return towards normal relatively quickly. Classically, this arises with diabetic ketoacidosis but might also occur in patients admitted to ICU after surgery while still under the effects of general anesthesia [4]. Due to this uncertainty and drawback, physicians are often interested in the use of newly or clinically available predictive biomarkers that are objectively, rapidly, cost-effectively measurable, respond to clinical recovery, and add relevant, reliable, and real-time information [5].

As one of the major contributors for the all-cause mortality, systematic inflammatory response (SIR) is the common pathophysiological reaction in the critically ill patients $[6,7]$. Markers of the SIR syndrome (SIRS), including CRP and PCT, as well as white blood cell count (WBC) have been shown to be prognostic of survival in patients in a variety of cancers [8-13]. However, the relationships between early CRP, PCT and WBC count at ICU admission and the mortality of severe patients have not been fully validated. We therefore conducted a multicenter, prospective, observational study to examine the possible independent relationships between the blood concentrations of the abovementioned inflammatory markers at ICU admission and ICU mortality in critically ill adults. The ability of the independent inflammatory markers for mortality prediction was further assessed.

\section{Methods}

\section{Study design and setting}

The study was conducted from September 1, 2018, to August 1, 2019, in four general ICUs of tertiary care hospitals in the Guangdong Province, China, which were multidisciplinary ICUs admitting patients from all medical areas with a specialty in surgery, including cardiothoracic surgery. When admitted into the ICU, patients were assessed for inclusion in the study. The inclusion criteria were: 1) length of ICU stay more than $24 \mathrm{~h}$; 2) age over 15 years old; 3) informed consent signed. The exclusion criteria included any of the following: length of stay (LOS) in the ICU $<24 \mathrm{~h}$; patients with thyroid tumors (e.g., thyroid adenoma or thyroid carcinoma); and inability to provide informed consent or unavailability of a proxy for informed consent. The primary endpoint was all-cause ICU mortality. The protocol was approved by the Institutional Ethics Committee of each participating center and was performed according to the ethical standards of the Declaration of Helsinki. Written informed consent was obtained from each patient or their legal surrogates. The study was registered at http://www.chictr.org.cn/showproj.aspx?proj=29522 (ChiCTR1800017806).

\section{Laboratory measurements}

All of the biomarkers were measured in a central laboratory, and all of the samples were labeled using study identification numbers without personal identifiers or clinical conditions. PCT was measured by Elecsys BRAHMS PCT (Roche Diagnostics GmbH, Germany; normal range, $\leq 0.05 \mu \mathrm{g} / \mathrm{L}$ ). CRP was measured by an immunoenzyme analyzer (Hitachi 917, Tokyo, Japan; normal range, $\leq 5 \mathrm{mg} / \mathrm{L}$ ). WBC counts were measured using an XE4000i automatic hemocyte analyzer. Blood samples for the purpose of study were collected only within $1 \mathrm{~h}$ after ICU admission, and clinicians decided the time and frequency of testing according to the actual clinical situation during the ICU stay.

\section{Data collection}

In addition to PCT and CRP concentrations and WBC count, we also collected the demographic and clinical characteristics of each patient, including sex, age, treatment, preexisting chronic conditions, sepsis, Charlson score, source of admission, SOFA score, APACHE II score, and LOS in the ICU. ICU mortality data were collected by reviewing medical records in the in-hospital patient data management system. Sepsis was diagnosed according to the Surviving Sepsis Guidelines [4].

\section{Statistical analysis}

Continuous variables are expressed as the median (IQR) and were compared with the Mann-Whitney U test; categorical variables are expressed as numbers (\%) and were compared by the $\chi^{2}$ test or Fisher's exact test between 
the survival and nonsurvival groups. All analyses were 2tailed and conducted by SPSS for Windows (version 26.0; IBM, Chicago, IL, USA) and R Statistical Software (version 5.3.0). A $P$ value $<0.05$ was considered statistically significant.

Discrimination was evaluated using the area under the curve (AUROC) derived from the conventional receiver operating characteristic (ROC) curve. AUROC of $>0.5,>0.6,>0.70$ or $>0.80$ were considered poor, fair, satisfactory or good, respectively [12]. AUROCs, as a measure of classification accuracy, were further compared with or without CRP added to the APAC HE II score using the nonparametric approach of DeLong and Clarke-Pearson [14]. Univariate and multivariate logistic regression analyses were used to detect factors independently associated with nonsurvival. The optimal cut-off values for individual biomarkers were determined using Youden's index. To evaluate the utility of the biomarkers for risk classification, we determined the category-free net reclassification improvement (NRI) and the integrated discrimination improvement (IDI), as previously described $[15,16]$.

In consideration of the possibility that dichotomized cutoffs may not accurately capture the usefulness of PCT and CRP, sensitivity analysis was used to assess whether alternative cut points for these biomarkers were more appropriate. We used a restricted cubic spline function with 3 knots for PCT and CRP to allow nonlinearity as continuous predictors in a multivariable model $[12,17,18]$.

\section{Results}

Baseline characteristics and outcomes of the patients

Of the 1526 consecutive patients who were screened for inclusion in the study, 713 (46.7\%) were excluded, and 813 patients were enrolled in the analyses (Fig. 1). The baseline characteristics and outcomes of the patients are summarized in Table 1. The serum concentrations of PCT and CRP were significantly higher in nonsurvivors than in survivors (PCT: $0.97[0.23 ; 5.51]$ vs. $0.12[0 ; 1.02]$ $\mu \mathrm{g} / \mathrm{L}, P=0.000 ; \mathrm{CRP} 66.70[12.80 ; 140.00]$ vs. 11.95 [2.25; 56.15] $\mathrm{mg} / \mathrm{L}, P=0.000)$. APACHE II score, SOFA score, age, source of admission, treatments, sepsis, preexisting clinical conditions including hypertension, COPD, coronary disease, diabetes mellitus and chronic heart failure also differed significantly between the two groups. The median days of LOS in the ICU were 6 and 3 in the survival and nonsurvival groups, respectively (Table 1).

\section{Risk factor analyses}

In the univariate analyses, elevations in PCT concentrations (odds ratio [OR]: 1.010 [1.003; 1.018], $P=0.004$ ) and CRP concentrations (OR: 1.008 [1.006; 1.011], $P=$ $0.000)$ were associated with an increased risk of ICU mortality. Additionally, APACHE II score, age, sepsis, hypertension, diabetes mellitus, COPD, coronary disease, mechanical ventilation, RRT, use of norepinephrine and chronic heart failure also had this association, whereas WBC was not determined to be a risk factor (Table 2).

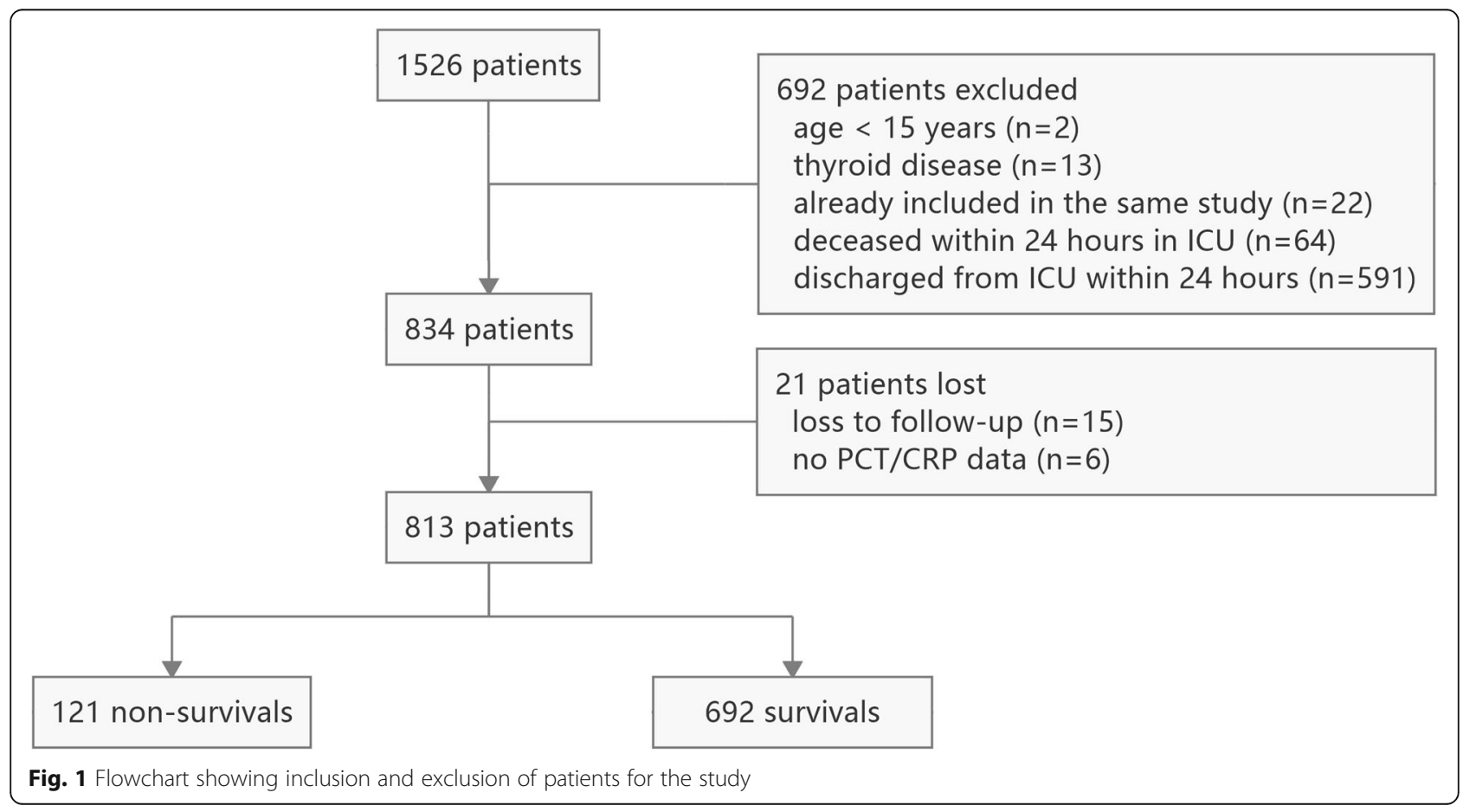


Table 1 Baseline characteristics and outcome

\begin{tabular}{|c|c|c|c|}
\hline Characteristics & $\begin{array}{l}\text { Non-survival } \\
(n=121)\end{array}$ & $\begin{array}{l}\text { Survival } \\
(n=692)\end{array}$ & $P$ value \\
\hline \multicolumn{4}{|l|}{ Demographic variables } \\
\hline Male, n (\%) & $69(57.02)$ & $376(54.34)$ & 0.584 \\
\hline Age (years), median (IQR) & $65(52-76)$ & $55(43.5-67)$ & $<0.001$ \\
\hline \multicolumn{4}{|l|}{ Preexisting clinical conditions } \\
\hline Hypertension, n (\%) & $48(39.67)$ & $200(28.90)$ & 0.015 \\
\hline COPD, n (\%) & $12(9.92)$ & $23(3.32)$ & 0.002 \\
\hline Coronary disease, n (\%) & $28(23.14)$ & $54(7.80)$ & $<0.001$ \\
\hline Diabetes mellitus, n (\%) & $25(20.66)$ & $86(12.43)$ & 0.016 \\
\hline Malignant tumor, n (\%) & $23(18.85)$ & $128(18.50)$ & 0.894 \\
\hline Chronic heart failure, n (\%) & $10(8.26)$ & $22(3.18)$ & 0.011 \\
\hline Chronic liver disease, n (\%) & $4(3.31)$ & $25(3.61)$ & 0.867 \\
\hline Charlson score, median (IQR) & $2(1-3)$ & $2(1-2)$ & 0.089 \\
\hline \multicolumn{4}{|l|}{ Source of Admission } \\
\hline Surgery, n (\%) & $58(47.93)$ & $522(75.43)$ & $<0.001$ \\
\hline Internal medicine, n (\%) & $30(24.79)$ & $44(6.36)$ & 0.002 \\
\hline Emergency, n (\%) & $34(28.10)$ & $127(18.35)$ & $<0.001$ \\
\hline \multicolumn{4}{|l|}{ Treatment } \\
\hline Mechanical ventilation, n (\%) & $97(80.17)$ & $465(67.20)$ & 0.005 \\
\hline RRT, n (\%) & $28(23.14)$ & $37(5.35)$ & $<0.001$ \\
\hline Use of corticosteroid, n (\%) & $32(26.45)$ & $148(21.39)$ & 0.217 \\
\hline Use of norepinephrine, n (\%) & $63(51.64)$ & $78(11.40)$ & $<0.001$ \\
\hline \multicolumn{4}{|l|}{ Inflammatory biomarkers, median (IQR) } \\
\hline $\mathrm{PCT}(\mu \mathrm{g} / \mathrm{L})$ & $0.97(0.23-5.51)$ & $0.12(0-1.02)$ & $<0.001$ \\
\hline $\mathrm{CRP}(\mathrm{mg} / \mathrm{L})$ & $66.70(12.80-140.00)$ & $11.95(2.25-56.15)$ & $<0.001$ \\
\hline WBC $\left(\times 10^{9} / \mathrm{L}\right)$ & $13.420(9.070-17.810)$ & $11.765(8.395-15.645)$ & 0.017 \\
\hline APACHEllscore, median (IQR) & $23(18-29)$ & $14(9-19)$ & $<0.001$ \\
\hline SOFA score, median (IQR) & $8(5-11)$ & $2(0-5)$ & $<0.001$ \\
\hline Sepsis, n (\%) & $82(67.77)$ & $223(32.23)$ & $<0.001$ \\
\hline \multicolumn{4}{|l|}{ Outcome } \\
\hline LOS of ICU (days), median (IQR) & $6(3-12)$ & $3(2-8)$ & $<0.001$ \\
\hline
\end{tabular}

APACHEII Acute Physiology and Chronic Health Evaluation II, COPD chronic obstructive pulmonary disease, CRP C-reactive protein, ICU intensive care unit, IQR interquartile range, LOS Length of stay, PCT procalcitonin, RRT Renal replacement therapy, SOFA Sequential Organ Failure Assessment, WBC, white blood count

Table 2 Predictive characteristics of admission markers for intensive care unit mortality

\begin{tabular}{|c|c|c|c|c|c|c|c|c|}
\hline \multirow[t]{2}{*}{ Markers } & \multicolumn{4}{|c|}{ Univariate Analysis } & \multicolumn{4}{|c|}{ Multivariate Analysis } \\
\hline & Cutoff & AUC $(95 \% \mathrm{Cl})$ & OR $(95 \% \mathrm{Cl})$ & $P$ value & Cutoff & AUC $(95 \% \mathrm{Cl})$ & OR (95\% Cl) & $P$ value \\
\hline APACHEll score & 20 & $0.816(0.777-0.854)$ & $1.174(1.139-1.210)$ & $<0.0001$ & $0.168^{\mathrm{a}}$ & $0.823(0.785-0.861)$ & $1.163(1.127-1.199)$ & $<0.0001$ \\
\hline CRP (mg/L) & 62.83 & $0.684(0.633-0.735)$ & $1.008(1.006-1.011)$ & 0.0037 & & & $2.145(1.343-3.427)$ & 0.003 \\
\hline PCT $(\mu \mathrm{g} / \mathrm{L})$ & 0.33 & $0.696(0.650-0.743)$ & $1.010(1.003-1.018)$ & 0.0039 & & & & 0.045 \\
\hline WBC $\left(\times 10^{9} / L\right)$ & 16.23 & $0.568(0.509-0.628)$ & $1.013(0.993-1.033)$ & 0.1977 & & & & 0.124 \\
\hline
\end{tabular}

APACHEII Acute Physiology and Chronic Health Evaluation II, AUC Area under the receiver operating characteristic curve, CRP C-reactive Protein, OR odds ratio, PCT Procalcitonin, WBC White blood cell count

${ }^{a}$ Cutoff point of the marker panels were the predicted probabilities corresponding to the best Youden's index generated from the multiple logistic regression model 


\section{ROC analyses}

ROC analysis revealed that, as continuous predictors, none of the inflammatory markers, including PCT and CRP concentrations and WBC count at ICU admission, were considered satisfactory in discriminating between survival and nonsurvival. The AUC-ROC value of PCT for discriminating ICU mortality was 0.696 (95\% CI: $0.650-0.743$ ), that of CRP was 0.684 (95\% CI: $0.633-0.735$ ), and that of WBC was 0.568 (95\% CI: 0.509-0.628). The same results were observed in the sepsis and nonsepsis subgroups (Supplementary Table 1). The optimal cut-off value of CRP for ICU mortality was $62.8 \mathrm{mg} / \mathrm{L}$, and the sensitivity and specificity were $52.1 \%(95 \% \mathrm{CI}, 42.8-61.2)$ and 76.7\% (95\% CI, 73.4-79.8), respectively. The positive and negative predictive values were $28.1 \%$ (95\% CI, $22.3-34.5)$ and $90.2 \% \quad(95 \% \quad \mathrm{CI}, \quad 87.5-94.2)$, respectively.

In the multivariable analysis, only APACHE II score (odds ratio [OR], 1.166; 95\% CI, 1.129-1.203; $P=0.000$ ) and CRP concentration $>62.8 \mathrm{mg} / \mathrm{L}(\mathrm{OR}, 2.145 ; 95 \% \mathrm{CI}$, $1.343-3.427 ; P=0.001$ ) were significantly associated with an increased risk of ICU mortality (Table 2), and this association did not seem to be significantly different between the sepsis and nonsepsis groups (Fig. 2). Moreover, adding the variable of CRP $>62.8 \mathrm{mg} / \mathrm{L}$ to the APACHE II score did not significantly increase the AUROC (Fig. 3).
Effect of CRP on the risk reclassification of ICU mortality Although CRP $>62.8 \mathrm{mg} / \mathrm{L}$ as a dichotomized variable did not significantly provide incremental value to the AUC of the APACHE II score, it markedly improved risk reclassification over the APACHE II score alone, displaying a category-free NRI of $0.556(P<0.0001)$ and an IDI of $0.013(P=0.0245)$ (Table 3$)$. Our analysis suggested that the addition of CRP to the APACHE II score considerably improved the prediction accuracy of ICU mortality, mainly due to increasing the correct predicted probabilities for without events.

\section{Sensitivity analyses}

In the sensitivity analysis using a restricted cubic spline function for PCT, there was no obvious nonlinearity between PCT and the risk of ICU mortality (Fig. 4A). However, CRP concentration $>62.8 \mathrm{mg} / \mathrm{L}$ was confirmed to be the optimal cut-off value in differentiating between patients with survival and those without survival (Fig. 4B). The sensitivity and specificity of using CRP $>62.8 \mathrm{mg} / \mathrm{L}$ as a cut-off point to predict ICU mortality were 0.521 (95\% CI, 0.43-0.72) and 0.767 (95\% CI, 0.55-0.87), respectively. The positive and negative predictive values were $0.281(95 \%$ $\mathrm{CI}, \quad 0.214-0.516)$ and 0.902 (95\% CI, 0.85-0.98), respectively.

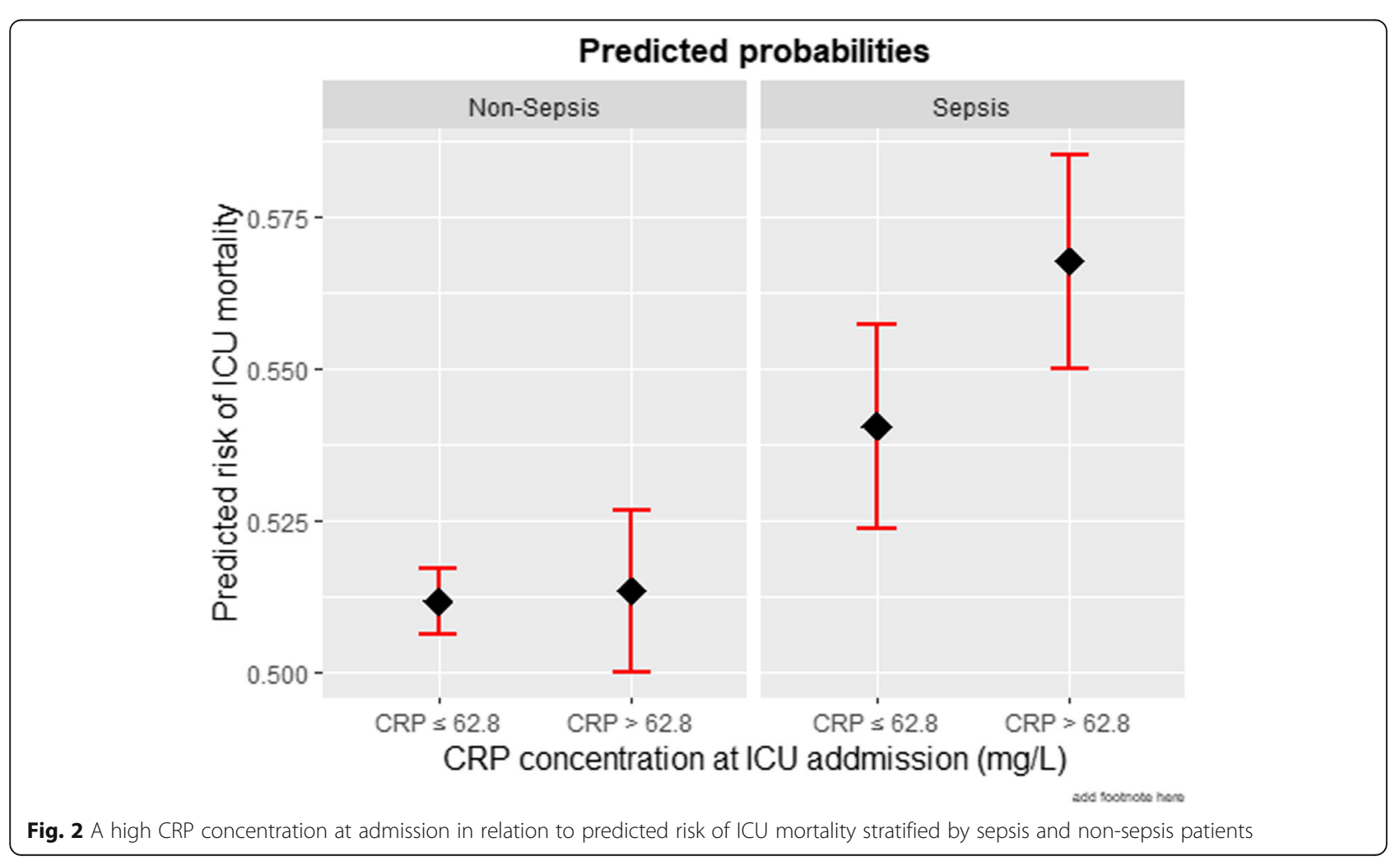




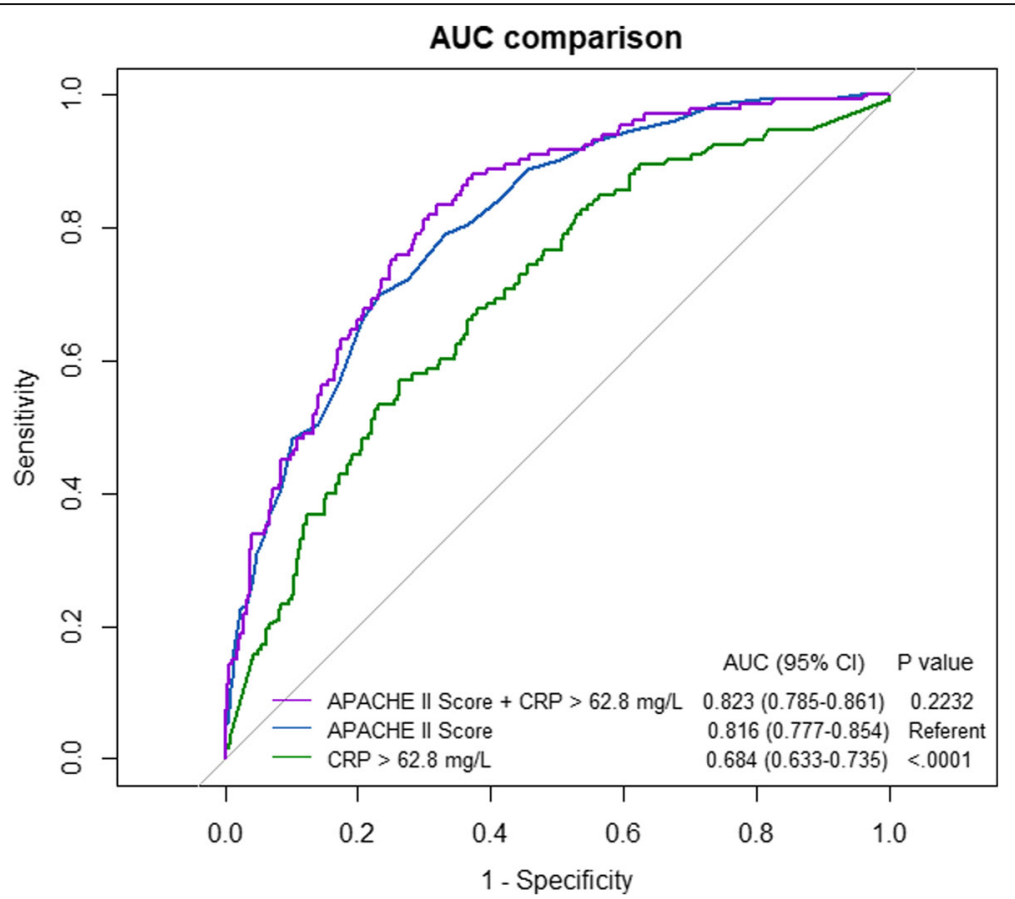

Fig. $3 \mathrm{ROC}$ analysis of CRP $>62.8 \mathrm{mg} / \mathrm{L}$ and APACHE II score biomarkers and their combinations for intensive care unit mortality prediction

\section{Discussion}

In our study of critically ill patients, at ICU admission, the serum concentrations of PCT and CRP and WBC count were significantly lower in survivors than in nonsurvivors, which is similar to the findings of previous studies $[12,19]$. As a continuous predictor, none of the inflammatory markers at ICU admission, including PCT concentrations, had sufficient discriminative ability for predicting ICU mortality. However, as a dichotomized variable, CRP $>62.8 \mathrm{mg} / \mathrm{L}$ at ICU admission was associated with an increased odds of ICU mortality regardless of whether the patient was septic or not. This association remained significant even after adjusting for the APACHE II score, WBC count, and PCT concentration. The findings have clinical implications that some clinically available inflammatory markers may be useful for helping clinicians assess the prognosis of patients in the ICU, which is worth further confirmation.

In clinical practice, PCT concentration is a wellestablished marker in septic patients [20-23]. However, this real-world study with a sizable sample did not provide evidence for the usefulness of PCT as a predictor of ICU mortality, and this negative association was not due to any nonlinear relationship between PCT and mortality. Although the PCT concentrations of nonsurvivors were significantly higher than those of survivors, the difference was not significant after adjusting for the APACHE II score, WBC count, and CRP concentration, and its AUC-ROC curve was not satisfactory. This result is not surprising since patients with different pathogens might have differently increased PCT values [24]. PCT elevation also occurs in nonsepsis states, such as postoperative conditions, cardiogenic shock or resuscitated cardiac arrest. In addition, PCT levels may be low in patients with viral infections, localized infections or early infections [25]. This negative finding may be due to the complicated reasons for ICU admission. On the other hand, even though the PCT concentration is increased in the serum of patients with bacterial infection, the half-life of PCT is short, and the maximum daily decrease under effective anti-infective therapy is 50\% [26]. Therefore, with infection control, the PCT concentration

Table $3 \mathrm{NRI}$ and IDI analyses for risk reclassification of ICU mortality

\begin{tabular}{|c|c|c|c|c|c|c|c|c|}
\hline \multirow[t]{2}{*}{ Models } & \multirow{2}{*}{$\begin{array}{l}\text { Category-free } \\
\text { NRI }(95 \% \mathrm{Cl})\end{array}$} & \multirow[t]{2}{*}{$P$ value } & \multicolumn{4}{|c|}{ Category-free NRI $(95 \% \mathrm{Cl})$} & \multirow[t]{2}{*}{ IDI (95\% CI) } & \multirow[t]{2}{*}{$P$ value } \\
\hline & & & With Event & $P$ value & Without Event & $P$ value & & \\
\hline APACHE I| score & Referent & & & & & & Referent & \\
\hline APACHE II score + CRP > $62.8 \mathrm{mg} / \mathrm{L}$ & $\begin{array}{l}0.556 \\
(0.3705-0.7484)\end{array}$ & $<.0001$ & $0.02(0.0123-0.0324)$ & 0.7851 & $0.53(0.435-0.7371)$ & $<.0001$ & $\begin{array}{l}0.013 \\
(0.008-0.024)\end{array}$ & 0.0245 \\
\hline
\end{tabular}

APACHE II Acute Physiology And Chronic Health Evaluation II, Cl confidence interval, CRP C-reactive protein, IDI integrated discrimination improvement; NRI Net reclassification index 

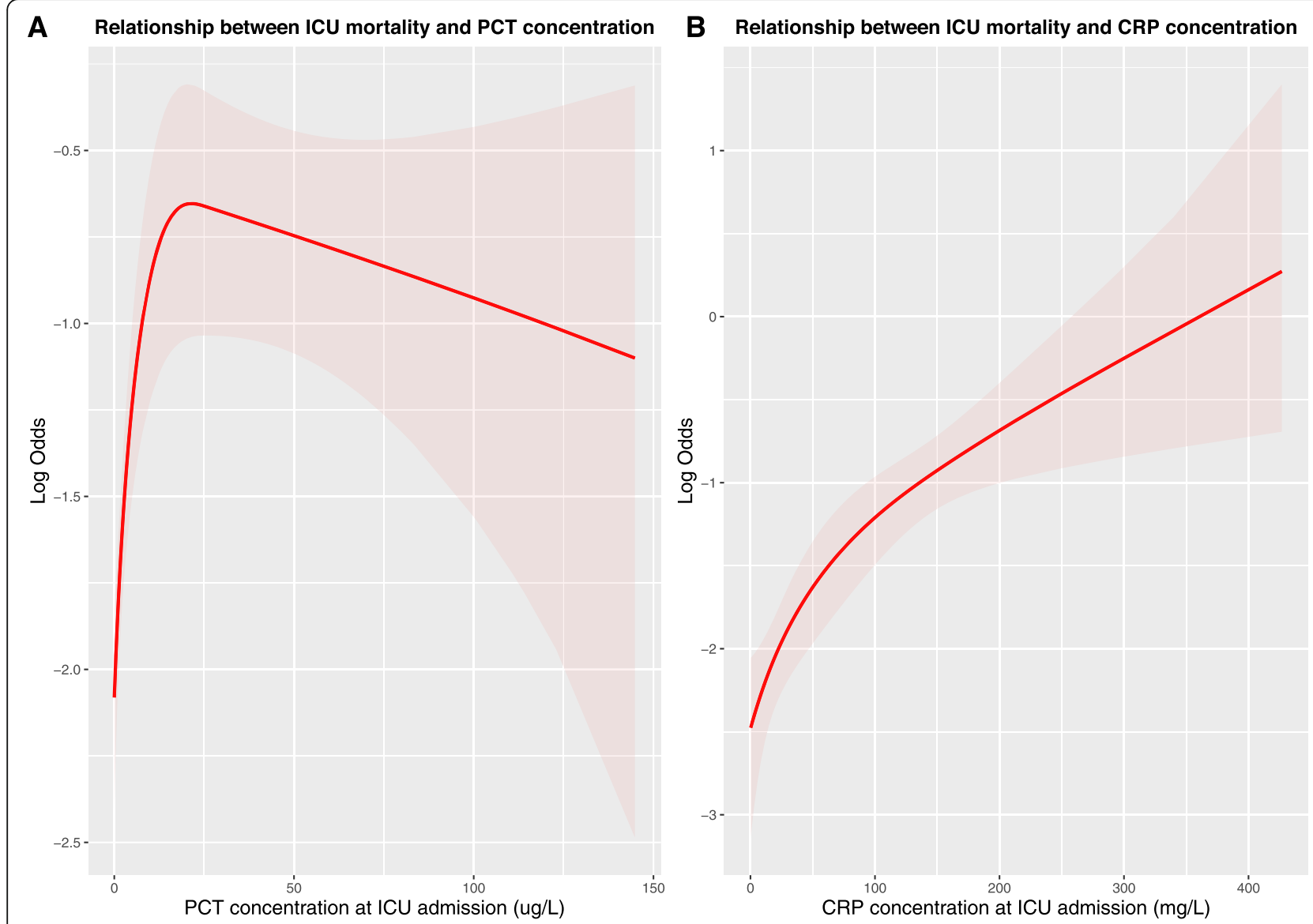

Fig. 4 Relationship between risk of ICU mortality and PCT (A) and CRP (B) concentrations at ICU admission, allowing PCT and CRP as a nonlinear continuous predictor using a restricted cubic spline 3-knot function while adjusting for the APACHEllscore, WBC, and CRP concentration. Shadow area show $95 \% \mathrm{Cl}$

decreases gradually, and the risk of death decreases accordingly. Otherwise, with the progression of infection, the PCT concentration continues to increase, and the risk of death also increases. This may be another reason why the discriminatory performance of PCT was poor in our population. This finding reminds us that more attention should be paid to the trend of PCT serum levels and not only one PCT value [20,23, 27]. Of course, the time frame between two consecutive samples needs further investigation.

CRP has been used for many years [28-30]. However, as it is difficult to differentiate sepsis from other nonsepsis causes of SIR, its specificity has been challenged [31, 32]. In the study, the CRP concentrations of nonsurvivors were significantly higher than those of survivors. However, its AUC-ROC curve was not satisfactory. As a dichotomized variable, CRP $>62.8 \mathrm{mg} / \mathrm{L}$ at ICU admission was associated with an increased odds of ICU mortality regardless of whether the patient was septic or not. This may be related to the lack of specificity of CRP in both sepsis and nonsepsis patients [31]. The findings suggested that even inflammation without infection may still be associated with ICU mortality. The negative predictive value of CRP showed that patients with a low CRP $(<62.8 \mathrm{mg} / \mathrm{L})$ at ICU admission had a low risk of ICU mortality. Therefore, in clinical practice, CRP may be useful for helping clinicians assess the prognosis of ICU patients.

In our study, APACHE II scores other than WBC count were independently associated with the outcome of nonsurvival. As the APACHE II score is a physiologically based system containing 12 physiological parameters, it is a useful prediction tool for hospital consequences, including mortality in critically ill patients [33]. Moreover, the APACHE II scoring system includes WBC count, and thus, pathophysiological changes after systemic insult could be illustrated comprehensively and systematically by the APACHE II scoring system. Therefore, this scoring system is thought to be superior to WBC count for the prediction of adverse outcomes [34]. 
Furthermore, we determined that the variable of CRP > $62.8 \mathrm{mg} / \mathrm{L}$ did not increase the prediction performance of the APACHE II score using the AUC-ROC comparison by the DeLong method. However, the AUC has recently been criticized for its insensitivity in model comparisons in which the baseline model has performed well [35]. Thus, 2 other measures have been proposed to capture the improvement in discrimination for nested models: integrated discrimination improvement and continuous net reclassification improvement. In the present study, we found that the dichotomized variable of CRP markedly improved risk reclassification over the APACHE II score, displaying a category-free NRI of 0.556 and an IDI of 0.013 . On the basis of these results, we concluded that the APACHE II score plus CRP is more promising in terms of improving predictive value than the APACHE II score alone.

There are advantages in this study. To the best of our knowledge, this is the largest study exploring the prognostic value of PCT at ICU admission for patients with and without sepsis. Moreover, it is a multicenter prospective study that allows the retrieval of real-world data with potentially better data quality than retrospective designs, and our results may be generalizable to other centers that have different cases. However, there are some limitations in the study. First, many studies $[36,37]$ revealed that distinct groups of pathogens and different foci of infection determined different PCT serum concentration. In this study, the specific information about infections were not analyzed. It was due to the incomplete data regarding the pathogens and foci of infection. In addition to pathogens, there are many other contributing factors like the source of ICU admission, which showed difference in the constitution of the two groups. Second, CRP at ICU admission was associated with ICU mortality, and this association did not seem to be different between septic patients and nonseptic patients, but the sensitivity and specificity were not perfect. Third, many confounders may still exist and potentially determine bias. For example, some preexisting clinical conditions (i.e., hypertension, diabetes mellitus, coronary disease, diabetes mellitus) presented high proportions in the non-survival than in the survival group. Thus, further studies, such as those combining multiple biomarkers, are essential for improving the prediction performance.

PCT concentration and WBC count at ICU admission were inadequate in their predictive ability of ICU mortality. As a dichotomized variable, CRP $>62.8 \mathrm{mg} / \mathrm{L}$ at ICU admission was associated with an increased odds of ICU mortality regardless of whether the patient was septic or not. The negative predictive value of CRP showed that patients with a low CRP $(<62.8 \mathrm{mg} / \mathrm{L})$ at ICU admission had a low risk of ICU mortality. Further studies, such as those combining multiple biomarkers, are essential for improving the prediction performance.

\section{Conclusions}

For inflammatory biomarkers at ICU admission, the concentration of CRP, and not PCT or WBC, can be an independent risk factor for ICU mortality, and CRP can improve risk reclassification for prognosis prediction.

\section{Supplementary Information}

The online version contains supplementary material available at https://doi. org/10.1186/s12871-020-01207-3.

Additional file 1: Supplementary Table 1 Area under the ROC curve for inflammatory markers and clinical scoring systems at ICU admission in discriminating ICU mortality.

Additional file 2.

\section{Abbreviations}

APACHE II: Acute Physiology and Chronic Health Evaluation II; AUC: Area under receiver operating characteristic curve; Cl: Confidence interval; CRP: Creactive protein; ICU: Intensive care unit; IQR: Interquartile range; OR: Odds ratio; PCT: Procalcitonin; ROC: Receiver operating characteristic curve; SD: Standard deviation; SIR: Systematic inflammatory response; WBC: White blood cell

\section{Acknowledgments}

The authors would like to thank all the doctors, nurses, technicians, and patients for their dedication to the study.

\section{Authors' contributions}

$\mathrm{CBC}$ designed the study, analyzed the data, and wrote the manuscript. $R Q$, $\mathrm{LHH}$ contributed to the study concept. $\mathrm{XL}$, CBC contributed to study coordination. RQ contributed to data collection. RQ and $\mathrm{LHH}$ did the statistical analysis. All authors contributed to data interpretation and approved the manuscript.

\section{Funding}

Chunbo Chen is currently receiving a grant (\#201803010058) from the Guangzhou Livelihood Science and Technology Project; Chunbo Chen is currently receiving a grant (No.DFJH2020028) under the major program of Summit Project, Guangdong Province High-level Hospital Construction Project of Guangdong Provincial People's Hospital, Guangdong Academy of Medical Sciences; Linhui Hu is currently receiving a grant (\#2020YJ01) from Emergent Science and Technology Project for Prevention and Treatment of Novel Coronavirus Pneumonia of Maoming City and High-level Hospital Construction Research Project of Maoming People's Hospital (\#zx2020017). The funding sources played no role in the design of the study and collection, analysis or interpretation of data, and played no role in the drafting, revision, or submission of the manuscript.

\section{Availability of data and materials}

The datasets used and/or analyzed during the current study are available from the corresponding author on reasonable request.

\section{Ethics approval and consent to participate}

All procedures performed in the trial were in accordance with the ethical standards of the ethics committee of all the participating centers (Ethics Committee of Huizhou Municipal Central Hospital; NO. LLBA201804A) and with the 1964 Helsinki Declaration and its later amendments or comparable ethical standards. Informed consent was written from all individual participants included in the study.

Consent for publication Not applicable.

Competing interests

The authors declare that they have no competing interests. 


\section{Author details}

The Second School of Clinical Medicine, Southern Medical University, Guangzhou 510515, Guangdong, China. 'Department of Critical Care Medicine, Huizhou Municipal Central Hospital, 41 North E'ling Road, Huizhou 516001, Guangdong, China. ${ }^{3}$ Department of Critical Care Medicine, Maoming People's Hospital, 101 Weimin Road, Maoming 525000, Guangdong, China. ${ }^{4}$ Clinical Research Center, Maoming People's Hospital, 101 Weimin Road, Maoming 525000, Guangdong, China. ${ }^{5}$ Department of Intensive Care Unit of Cardiovascular Surgery, Guangdong Cardiovascular Institute, Guangdong Provincial People's Hospital, Guangdong Academy of Medical Sciences, 96 Dongchuan Road, Guangzhou 510080, Guangdong, China. ${ }^{6}$ Department of Critical Care Medicine, Guangdong Provincial People's Hospital, Guangdong Academy of Medical Sciences, 106 Zhongshan Er Road, Guangzhou 510080, Guangdong, China. ${ }^{7}$ State Key Laboratory of Organ Failure Research, Guangdong Provincial Key Laboratory of Viral Hepatitis Research, Department of Infectious Diseases, Nanfang Hospital, Southern Medical University, 1838 Guangzhou Avenue North, Guangzhou, Guangdong, China. ${ }^{8}$ Department of Critical Care Medicine, Maoming People's Hospital Affiliated to Southern Medical University, 101 Weimin Road, Maoming 525000, Guangdong, China.

Received: 11 September 2020 Accepted: 17 November 2020

\section{Published online: 23 November 2020}

\section{References}

1. Kuzniewicz MW, Vasilevskis EE, Lane R, Dean ML, Trivedi NG, Rennie DJ, Clay T, Kotler PL, Dudley RA. Variation in ICU risk-adjusted mortality: impact of methods of assessment and potential confounders. Chest. 2008;133(6):1319-27.

2. Raith EP, Udy AA, Bailey M, McGloughlin S, Maclsaac C, Bellomo R, Pilcher DV. Prognostic accuracy of the SOFA score, SIRS criteria, and qSOFA score for in-hospital mortality among adults with suspected infection admitted to the intensive care unit. Jama. 2017;317(3):290-300.

3. Singer M, Deutschman CS, Seymour CW, Shankar-Hari M, Annane D, Bauer M, Bellomo R, Bernard GR, Chiche JD, Coopersmith CM, et al. The third international consensus definitions for Sepsis and septic shock (Sepsis-3). Jama. 2016;315(8):801-10.

4. Dellinger RP, Levy MM, Rhodes A, Annane D, Gerlach H, Opal SM, Sevransky JE, Sprung CL, Douglas IS, Jaeschke R, et al. Surviving Sepsis campaign: international guidelines for management of severe sepsis and septic shock, 2012. Intensive Care Med. 2013;39(2):165-228.

5. Cohen MJ, Grossman AD, Morabito D, Knudson MM, Butte AJ, Manley GT. Identification of complex metabolic states in critically injured patients using bioinformatic cluster analysis. Crit Care. 2010;14(1):R10.

6. Brun-Buisson C. The epidemiology of the systemic inflammatory response. Intensive Care Med. 2000;26(Suppl 1):S64-74.

7. Pantzaris ND, Platanaki C, Pierrako C, Karamouzos V, Velissaris D. Neutrophilto-lymphocyte ratio relation to Sepsis severity scores and inflammatory biomarkers in patients with community-acquired pneumonia: a case series. J Transl Int Med. 2018;6(1):43-6.

8. McMillan DC. The systemic inflammation-based Glasgow prognostic score: a decade of experience in patients with cancer. Cancer Treat Rev. 2013;39(5): 534-40.

9. Ho KM, Lee KY, Dobb GJ, Webb SA. C-reactive protein concentration as a predictor of in-hospital mortality after ICU discharge: a prospective cohort study. Intensive Care Med. 2008;34(3):481-7.

10. Zhang $\mathrm{Z}, \mathrm{Ni} \mathrm{H}$. C-reactive protein as a predictor of mortality in critically ill patients: a meta-analysis and systematic review. Anaesth Intensive Care. 2011;39(5):854-61.

11. Aygun F. Procalcitonin value is an early prognostic factor related to mortality in admission to pediatric intensive care unit. Crit Care Res Pract. 2018;2018:9238947.

12. Zhou G, Ho KM. Procalcitonin concentrations as a predictor of unexpected readmission and mortality after intensive care unit discharge: a retrospective cohort study. J Crit Care. 2016;33:240-4.

13. Erlinger TP, Muntner P, Helzlsouer KJ. WBC count and the risk of cancer mortality in a national sample of U.S. adults: results from the second National Health and nutrition examination survey mortality study. Cancer Epidemiol Biomark Prev. 2004;13(6):1052-6.

14. DeLong ER, DeLong DM, Clarke-Pearson DL. Comparing the areas under two or more correlated receiver operating characteristic curves: a nonparametric approach. Biometrics. 1988;44(3):837-45.

15. Cook NR. Statistical evaluation of prognostic versus diagnostic models: beyond the ROC curve. Clin Chem. 2008;54(1):17-23.
16. Pencina MJ, D'Agostino RB Sr, D'Agostino RB Jr, Vasan RS. Evaluating the added predictive ability of a new marker: from area under the ROC curve to reclassification and beyond. Stat Med. 2008;27(2):157-72 discussion 207-112.

17. Ho KM, Knuiman M, Finn J, Webb SA. Estimating long-term survival of critically ill patients: the PREDICT model. PLoS One. 2008;3(9):e3226.

18. Gauthier J, Wu QV, Gooley TA. Cubic splines to model relationships between continuous variables and outcomes: a guide for clinicians. Bone Marrow Transplant. 2020;55(4):675-80.

19. Chirapongsathorn S, Bunraksa W, Chaiprasert A, Punpanich D, Supasyndh O, Kamath PS. Adding C-reactive protein and procalcitonin to the model of end-stage liver disease score improves mortality prediction in patients with complications of cirrhosis. J Gastroenterol Hepatol. 2018;33(3):726-32.

20. Bartoletti M, Antonelli M, Bruno Blasi FA, Casagranda I, Chieregato A, Fumagalli R, Girardis M, Pieralli F, Plebani M, Rossolini GM, et al. Procalcitonin-guided antibiotic therapy: an expert consensus. Clin Chem Lab Med. 2018;56(8):1223-9.

21. Wu CC, Lan HM, Han ST, Chaou CH, Yeh CF, Liu SH, Li CH, Blaney GN 3rd, Liu ZY, Chen KF. Comparison of diagnostic accuracy in sepsis between presepsin, procalcitonin, and C-reactive protein: a systematic review and meta-analysis. Ann Intensive Care. 2017;7(1):91.

22. Gao X, Liu Y, Xu F, Lin S, Song Z, Duan J, Yin Y, Cao J. Assessment of apoptosis inhibitor of macrophage/CD5L as a biomarker to predict mortality in the critically ill with Sepsis. Chest. 2019;156(4):696-705.

23. Schuetz $P$, Birkhahn R, Sherwin R, Jones AE, Singer A, Kline JA, Runyon MS, Self WH, Courtney DM, Nowak RM, et al. Serial Procalcitonin predicts mortality in severe Sepsis patients: results from the multicenter Procalcitonin MOnitoring SEpsis (MOSES) study. Crit Care Med. 2017;45(5): 781-9.

24. Leli C, Ferranti M, Moretti A, Al Dhahab ZS, Cenci E, Mencacci A. Procalcitonin levels in gram-positive, gram-negative, and fungal bloodstream infections. Dis Markers. 2015;2015:701480.

25. Hausfater $P$, Juillien G, Madonna-Py B, Haroche J, Bernard M, Riou B. Serum procalcitonin measurement as diagnostic and prognostic marker in febrile adult patients presenting to the emergency department. Crit Care. 2007;11(3):R60

26. Schuetz $P$, Albrich W, Mueller B. Procalcitonin for diagnosis of infection and guide to antibiotic decisions: past, present and future. BMC Med. 2011;9:107.

27. Yu H, Qi Z, Hang C, Fang Y, Shao R, Li C. Evaluating the value of dynamic procalcitonin and presepsin measurements for patients with severe sepsis. Am J Emerg Med. 2017;35(6):835-41.

28. Schmit $X$, Vincent JL. The time course of blood C-reactive protein concentrations in relation to the response to initial antimicrobial therapy in patients with sepsis. Infection. 2008;36(3):213-9.

29. Pierrakos C, Vincent JL. Sepsis biomarkers: a review. Crit Care. 2010;14(1):R15.

30. Weidhase L, Wellhöfer $D$, Schulze G, Kaiser T, Drogies T, Wurst U, Petros S. Is Interleukin-6 a better predictor of successful antibiotic therapy than procalcitonin and C-reactive protein? A single center study in critically ill adults. BMC Infect Dis. 2019;19(1):150.

31. Clyne B, Olshaker JS. The C-reactive protein. J Emerg Med. 1999;17(6):1019-25.

32. Velissaris D, Pantzaris ND, Skroumpelou A, Polychronopoulos P, Karamouzos V, Pierrakos C, Gogos C, Karanikolas M. Electroencephalographic abnormalities in Sepsis patients in correlation to the calculated prognostic scores: a case series. J Transl Int Med. 2018;6(4):176-80.

33. Siew ED, Ware LB, Gebretsadik T, Shintani A, Moons KG, Wickersham N, Bossert F, Ikizler TA. Urine neutrophil gelatinase-associated lipocalin moderately predicts acute kidney injury in critically ill adults. J Am Soc Nephrol. 2009;20(8):1823-32.

34. Caronna JJ, Stübgen JP. Predicting mortality in intensive care unit patients with stroke. Crit Care Med. 2000;28(5):1656-7.

35. Pencina MJ, D'Agostino RB, Pencina KM, Janssens AC, Greenland P. Interpreting incremental value of markers added to risk prediction models. Am J Epidemiol. 2012;176(6):473-81.

36. Cortegiani A, Misseri G, Ippolito M, Bassetti M, Giarratano A, Martin-Loeches I, Einav S. Procalcitonin levels in candidemia versus bacteremia: a systematic review. Crit Care. 2019;23(1):190.

37. Thomas-Rüddel DO, Poidinger B, Kott M, Weiss M, Reinhart K. Bloos F, for the Msg: influence of pathogen and focus of infection on procalcitonin values in sepsis patients with bacteremia or candidemia. Crit Care. 2018;22(1):128.

\section{Publisher's Note}

Springer Nature remains neutral with regard to jurisdictional claims in published maps and institutional affiliations. 